Discussion We found that even in an area which has achieved high screening coverage, few people were re-screened. However, positive cases are more likely to be retested than negatives. This detailed county level analysis will provide information to policy makers to develop a long-term, sustainable and effective screening strategy.

\section{P5-S7.08 ASSESSMENT OF CHLAMYDIA TESTING COVERAGE USING HEDIS DATA: USA, 2009}

doi:10.1136/sextrans-2011-050108.603

G Tao, K Hoover, R Romaguera, C Kent. CDC, Atlanta, USA

Background In the USA, chlamydia screening of all sexually active women aged $\leq 25$ years is recommended, but screening rates are low. The Healthcare Effectiveness Data and Information Set (HEDIS) performance measure has tracked the time trend in screening since 2000. It has been found that among sexually active women aged $15-25$ years, chlamydia testing rates increased from $25.3 \%$ to $41.6 \%$ during 2000-2007. While rates have increased, they remain suboptimal, and it is unknown whether testing rates differ significantly between 2007 and 2009 or by the type of health plan.

Methods The 2009 HEDIS chlamydia testing dataset was analysed to estimate the annual coverage among sexually active women aged $15-25$ years who were enrolled in commercial health plans. Sexually active women were defined as those who had a claim or visit for pregnancy, contraception, cervical cancer screening, or STD diagnosis, screening, or treatment. A woman was counted as having a test if she had a claim or healthcare visit for any chlamydia test. Health plan types included in the HEDIS data are health maintenance organisations (HMOs), preferred provider organisation (PPO), point of service (POS), and any combination of $\mathrm{HMO}, \mathrm{PPO}$, and POS.

Results Of 2.4 million sexually active women, $54.5 \%$ were enrolled in $\mathrm{PPO}, 28.7 \%$ in combined $\mathrm{HMO} / \mathrm{POSs}, 13.8 \%$ in $\mathrm{HMOs}, 2.7 \%$ in combined $\mathrm{HMO} / \mathrm{POS} / \mathrm{PPO}$, and $0.3 \%$ in POSs. The overall annual chlamydia testing rate was $44.2 \%$ and significantly differed by plan type: $56.1 \%$ in $\mathrm{HMOs}, 45.7 \%$ in combined $\mathrm{HMO} / \mathrm{POSs}, 44.6 \%$ in POSs, 41.2 in combined $\mathrm{HMO} / \mathrm{POS} / \mathrm{PPO}$, and $40.5 \%$ in PPOs.

Conclusions The annual chlamydia testing rate continued to increase between 2007 and 2009 (41.6\% to 44.2\%). However, the testing rate remains suboptimal, suggesting that additional interventions are needed to further increase testing rates. The highest testing rates were found in $\mathrm{HMOs}$, healthcare settings that have interventions in place to promote provision of recommended healthcare services. The 2010 Affordable Care Act requires insurance plans cover preventive services such as chlamydia screening without patient cost sharing, and has the potential to increase chlamydia screening coverage throughout the US healthcare system. In light of these changes, it is important to continue to monitor chlamydia testing practices and to overcome barriers to testing.

\section{P5-S7.09 IS ABANDONING URETHRAL SMEAR MICROSCOPY FOR THE DETECTION OF NON-GONOCOCCAL NON-CHLAMYDIAL URETHRITIS IN ASYMPTOMATIC MEN A COST EFFECTIVE STRATEGY?}

doi:10.1136/sextrans-2011-050108.604

${ }^{1} \mathrm{O}$ Caffrey, ${ }^{2} \mathrm{~J}$ Saunders, ${ }^{2} \mathrm{C}$ Estcourt, ${ }^{3} \mathrm{R}$ Birger, ${ }^{3} \mathrm{P}$ White, ${ }^{1} \mathrm{~T}$ Roberts. ${ }^{1}$ University of Birmingham, Birmingham, UK; ${ }^{2}$ Oueen Mary University of London, London, UK; ${ }^{3}$ Imperial College London London, UK

Background Asymptomatic non-gonococcal non-chlamydial urethritis (NCNGU) is common and can only be diagnosed by urethral smear microscopy. UK guidelines no longer recommend urethral smear microscopy in asymptomatic men, leaving men with this condition and their sexual partners untreated. The clinical and economic significance of this is unclear. We do not know if the costs of microscopy screening for asymptomatic NCNGU outweigh any future health benefits. We performed a model based economic evaluation to compare a screening strategy which includes microscopy to one which omits microscopy in asymptomatic men. Methods The economic model was from the perspective of the UK health service and so only direct medical costs and outcomes are included. A hypothetical cohort of asymptomatic men who present at sexual health clinics, or at primary care is assumed to have Chlamydia trachomatis, Neisseria gonorrhoeae or NCNGU. In the economic model, untreated infections were taken into account for patients and their partners (pelvic inflammatory disease and tubal factor infertility in female partners). Probabilities and frequencies of these health events were informed by the outputs of a mathematical transmission model which used data from literature and national databases. A bottom-up costing estimated the cost for microscopy screening, while other cost inputs were sourced from published literature and online reference manuals for the economic evaluation. Appropriate sensitivity analyses were conducted to test the baseline results.

Results Currently, there are no robust quality-adjusted-life-year (OALY) data to value STI outcomes. Consequently, results will be presented as cost per major outcome avoided and cost per infection avoided, where major outcome averted refers to PID, infertility and tubal factor infertility. Incremental cost-effective ratios will be interpreted based on accepted precedents.

Conclusions The UK's current financial restraint has increased the importance of allocating resources based on need and value-formoney. This in turn, requires the UK health service to eliminate ineffective and inefficient services to create additional source of funding. Results from this study will indicate whether microscopy testing for asymptomatic men is good use of public money and whether funding for the service should be maintained.

\section{P5-S7.10 CHLAMYDIA SCREENING COVERAGE AMONG AMERICAN INDIAN AND ALASKA NATIVE WOMEN IN THE USA}

doi:10.1136/sextrans-2011-050108.605

S Tulloch, M Taylor. Centers for Disease Control \& Prevention, Albuquerque, USA

Background Racial and ethnic minorities are often disproportionately affected by health disparities. In 2009, American Indian and Alaska Natives (AI/AN) had the 2nd highest rates of chlamydia in the USA. In an effort to eliminate health inequities and reduce the burden of disease, the US Centers for Disease Control and Prevention (CDC) recommends annual chlamydia screening for all sexually active women $<25$ while reducing screening among older less at-risk individuals. We sought to assess and describe geographic regional differences in how recommendations have been adopted across Indian Country to identify what opportunities exist for improving care within the Indian Health Service (IHS) National STD Program's Stop Chlamydia Project, a screening program that partners with IHS/Tribal/Urban Indian health centers (I/T/U) to enhance and expand chlamydia screening among AI/AN

Methods We calculated chlamydia screening coverage rates and associated per cent positivity for women $<25$ and $>26$ years of age screened through sites participating in the Stop Chlamydia Project by geographic region. Screening coverage was calculated using the 2009 Indian Health Services facility-level user population estimates as denominator values.

Results Screening coverage among AI/AN women tested in Stop CT Project sites varied across geographic regions. Among women $<25$, rates ranged from $50.8 \%$ (Alaska) to $6.8 \%$ (California). Chlamydia 